\title{
Un microcosme musical. Les chants des Xetá du Brésil
}

\section{Desidèrio Aytai}

Traducteur : Isabelle Schulte-Tenckhoff

\section{(2) OpenEdition Journals}

Édition électronique

URL : http://journals.openedition.org/ethnomusicologie/1591

ISSN : 2235-7688

Éditeur

ADEM - Ateliers d'ethnomusicologie

\section{Édition imprimée}

Date de publication : 1 octobre 1991

Pagination : 189-206

ISBN : 978-2-8257-0431-8

ISSN : 1662-372X

Référence électronique

Desidèrio Aytai, « Un microcosme musical. Les chants des Xetá du Brésil », Cahiers d'ethnomusicologie [En ligne], 4 | 1991, mis en ligne le 01 janvier 2012, consulté le 19 avril 2019. URL : http://

journals.openedition.org/ethnomusicologie/1591 


\title{
UN MICROCOSME MUSICAL Les chants des Xetá du Brésil *
}

\author{
Desidério Aytai
}

L'histoire écrite des Xetá ne couvre qu'une quarantaine d'années: c'est vers 1949 que les premiers témoignages sur ce groupe établi dans la Serra dos Dourados (État de Paraná, Brésil) parviennent aux autorités (LamingEmperaire 1964: 263-264). En 1952, deux garçons xetá sont capturés et emmenés à Curitiba, capitale de l'État, pour être assimilés à la vie moderne brésilienne. Vers 1955, tandis que des liens se nouent avec des Xetá se faisant embaucher comme ouvriers agricoles, une expédition organisée conjointement par l'Université fédérale de Paraná et le Service de Protection de l'Indien est lancée pour contacter définitivement ce groupe. Par la suite, d'autres expéditions pénètrent dans la Serra dos Dourados et établissent des relations permanentes avec des groupuscules de Xetá qui mènent une vie plutôt simple, fondée sur une technologie de l'âge de pierre.

L'Université fédérale de Paraná s'est efforcée de préserver le plus d'informations possible sur la culture des Xetá, en entreprenant des études ethnologiques et linguistiques, en constituant des collections d'objets, en documentant par la photographie et le film divers aspects de leur mode de vie, ainsi qu'en enregistrant leur musique. Les résultats de ces études ont été partiellement publiés, et les collections d'objets et de documents sont actuellement conservées à l'Université. Parmi ces témoignages figurent cinq bandes magnétiques affichées «Matériaux xetá », qui nous ont été transmises avec l'autorisation de les copier, d'en étudier le contenu et de publier toute information d'intérêt scientifique s'y rapportant. Quelques autres bandes, probablement différentes de celles en notre possession, sont entre les mains de particuliers qui jusqu'ici n'en ont pas autorisé l'étude.

Les Xetá risquent l'extermination physique, et leur culture en tant qu'unité fonctionnelle a totalement disparu. Nous espérons contribuer à l'ethnologie d'urgence en publiant autant de données que possible sur leur musique.

D'une durée totale de 90 minutes environ, les enregistrements de la musique vocale et instrumentale des Xetá sont dans l'ensemble d'une assez bonne qualité, mais pas d'une fidélité qui permettrait d'entreprendre des études linguistiques et, en particulier, phonétiques.

Traduit de l'anglais par Isabelle Schulte-Tenckhoff. 
La première transcription donne le premier chant de la collection, dit Chant $d u$ vautour, enregistré le 27 janvier 1961 et interprété par un informateur du nom d'Arigã. Nous savons peu de choses sur cet homme. Sa technique vocale est très particulière, d'un type jamais rencontré parmi la douzaine de groupes amérindiens du Brésil dont la musique nous est familière. C'est un falsetto aigu exigeant de toute évidence beaucoup d'énergie et constituant une rude épreuve pour les cordes vocales: l'interprète tousse souvent et s'éclaircit la voix, même en chantant. Ce style vocal est difficile à décrire précisément. Il semblerait que le chanteur s'efforce d'imiter un instrument de musique, peut-être une flûte. D'autre part, n'étant pas familier avec ce style, il nous est difficile de détecter une différence quelconque entre les voix de deux chanteurs: on ne peut se défaire de l'impression que l'objectif de cette technique consiste à éliminer les différences vocales individuelles, la technique vocale des trois interprètes enregistrés étant quasiment identique.

Quant aux autres aspects de la musique des Xetá, l'observateur non indien est également frappé par cette aversion étonnante pour tout écart de l'habitus musical. L'échelle est tritonique, et les intervalles sont de deux demi-tons et deux demi-tons respectivement. L'échelle de quatre demi-tons est plutôt restreinte par comparaison avec la musique des Xavante, Bororo, Mamaindê, Halotesu, Wasusu, Pareci, Karajá et Guarani, que nous avons également eu l'occasion de transcrire. Mais même dans ce petit univers musical, le chanteur, qui est probablement aussi le compositeur, semble craindre des intervalles plus grands: sur les 278 sons de la transcription $\mathrm{N}^{\circ} 1$, on ne rencontre qu'un seul intervalle de quatre demi-tons (dans la mesure 33). Or, en comparant ce passage avec les passages correspondants de la structure strophique, on remarque immédiatement que cet intervalle est en réalité l'abrègement de deux sauts consécutifs de deux demi-tons chacun, le ré intermédiaire ayant été supprimé.

$\mathrm{Vu}$ ces limites étroites, il est malaisé de parler d'une ligne mélodique dont on voit d'ailleurs difficilement comment elle pourrait être autre chose qu'une légère ondulation. Le tempo est vif, allegro assai m 144, le tempo interne atteignant, selon Kolinski (1959: 45), un chiffre assez élevé, soit 172. La pièce est organisée selon un mètre simple - 2/4 - qui n'est cependant pas suivi rigoureusement (rubato), et elle possède une structure strophique dont les parties correspondantes montrent des modifications à peine perceptibles. A la première écoute, l'auditeur non averti croit entendre de nombreuses reprises de la même formule mélodique. Seule une transcription minutieuse permet de percevoir les infimes modulations qui semblent revêtir une importance cruciale pour l'auditeur xetá.

Il convient de s'arrêter brièvement sur le texte constitué de «syllabes inintelligibles » apparemment dépourvues de valeur sémantique. Cette impression n'est cependant fondée qu'en partie, si l'on se réfère à la musique d'autres groupes amérindiens où pareilles syllabes revêtent parfois une signification affective.

La deuxième pièce enregistrée le même jour est une version du Chant $d u$ vautour, interprétée par Ajatucã, le chef du groupe. Sa voix, plus rauque 
encore que celle d'Arigã, adopte la même technique de falsetto: Ajatucã a dû entendre le chant d'Arigã au cours de la séance précédente d'enregistrement, ce qui explique pourquoi il le reprend exactement à la même hauteur et avec la même tonalité. Or, en notant ce chant, nous nous sommes heurtés à notre tour à la difficulté qu'affronte bien souvent l'ethnomusicologue qui entreprend la transcription des chants indiens d'Amérique du Sud: à partir de la mesure 10, la hauteur du son augmente imperceptiblement d'abord, l'écart se chiffrant presque à un demi-ton à la fin de la pièce. Au lieu de marquer la modification de la hauteur pour chaque note individuelle, nous nous bornons cependant à ne l'indiquer qu'au début de la transcription: ayant rencontré le même phénomène dans d'autres musiques amérindiennes - xavante et karajá - nous avons constaté son caractère involontaire et aléatoire. Il arrive que le même interprète exécute le même chant à une hauteur précise quelques jours plus tard, alors qu'un autre n'altère pas du tout la hauteur de son dans un même morceau. C'est pourquoi nous renonçons à aborder ici la question de l'altération délibérée de la hauteur du son, mais non pas, en revanche, l'incapacité du chanteur de rester dans la même tonalité ni d'ailleurs son désintérêt à respecter la hauteur, comme le prescrit une règle fondamentale des genres musicaux fondés sur le jeu d'instruments mélodiques accordés, mais peu pertinente pour les genres purement vocaux.

A première vue, il est difficile de décider si les transcriptions $\mathrm{N}^{\text {os }} 1$ et 2 contiennent le même chant ou, ce qui serait également possible, deux pièces différentes. Or leurs différences n'en sont pas moins apparentes: la première transcription comporte 210 pulsations, la deuxième 151 . Le premier chant peut être divisé en huit phrases musicales, le deuxième en dix. Ces divisions sont indiquées dans nos transcriptions par des chiffres entourés d'un cercle. Sans considérer quelques différences insignifiantes - généralement de brève durée - nous constatons que dans la transcription $\mathrm{N}^{\circ} 1$, les phrases $1,2,3$ et 4 sont différentes, alors que 5, 6, 7 et 8 sont toutes identiques à 4. Ainsi la transcription $\mathrm{N}^{\mathrm{o}} 1$ contient-t-elle quatre phrases musicales.

Quant à la transcription $\mathrm{N}^{\mathrm{o}} 2$, nous obtenons le tableau suivant: les phrases 1, 2, 3, 4 et 5 sont identiques, mais différentes des phrases 6, 7, 8, 9 et 10, ellesmêmes identiques. Les deux premières lignes en fig. 1 schématisent cette relation, les phrases identiques étant reliées entre elles par un trait ininterrompu.

Alors que l'auditeur non averti ne percevrait pas de différences entre les deux pièces, celle-ci apparaît clairement dans l'enchaînement des phrases musicales de la fig. 1. Par conséquent, seule une analyse détaillée révèle ce qui ne resterait perceptible qu'aux familiers du microcosme musical des Xetá.

Afin de donner une base plus solide à notre comparaison, considérons la transcription d'un troisième Chant du vautour interprété cette fois-ci par Tonca «dans la voix» ou «d'après la voix» de son père. Pareille référence à l'interprète originel est significative. Les enregistrements comprennent une quantité d'autres Chants du vautour, dont la plupart sont interprétés «dans la voix» de quelqu'un, par exemple: «dans la voix du frère de sa femme» ou celle «de son frère défunt», ou encore celle d'un personnage appelé Koñãtxagui. Tout cela 


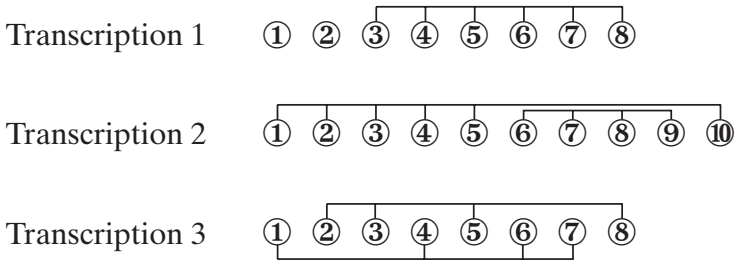

Fig. 1.

semble indiquer que de nombreux individus ont interprété le Chant du vautour, et chacun d'une manière légèrement différente.

Le chant interprété par Tonca est donné dans la transcription $\mathrm{N}^{\circ} 3$. Son échelle est plus réduite que celle des deux chants précédents, puisqu'elle se limite à deux intervalles de deux tons et d'un demi-ton respectivement. Il est constitué de huit phrases musicales dont 1, 4, 6 et 7 d'une part, 2, 3, 5 et 8 d'autre part sont identiques, comme l'indique la troisième portée de la fig. 1 . Aucune de ces phrases ne coïncide avec celles des deux premières transcriptions. Comme dans les deux chants précédents, la mélodie commence par une fondamentale qui est aussi la première et la dernière note de chaque phrase musicale.

Les trois transcriptions font apparaître plus ou moins la même structure

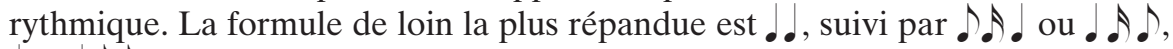
det..$\AA$. Le nombre de pulsations par phrase musicale varie selon les chants.

La transcription $\mathrm{N}^{\mathrm{o}} 1$ comporte des phrases de 13,14 et 15 temps, le transcription $\mathrm{N}^{\circ}$ 2, de 14 et 15 temps, enfin le transcription $\mathrm{N}^{\circ} 3$, de 7 et 14 temps. Quant au texte des trois chants, s'il nous est impossible d'affirmer s'il est constitué de «syllabes inintelligibles», il n'en correspond pas moins à la hauteur des sons, comme l'illustre la liste suivante: ${ }^{1}$

\begin{tabular}{|c|c|c|}
\hline & Son & Syllabes \\
\hline Transcription $\mathrm{N}^{\mathrm{o}} 1$ : & $\begin{array}{l}\text { grave } \\
\text { medium } \\
\text { aigu }\end{array}$ & $\begin{array}{l}\text { é è hé } \\
\text { é è hé hè } \\
\text { é è hé hè hi }\end{array}$ \\
\hline Transcription $\mathrm{N}^{\mathrm{o}} 2$ : & $\begin{array}{l}\text { grave } \\
\text { medium } \\
\text { aigu }\end{array}$ & $\begin{array}{l}\text { a é è } \\
\text { a é è hé hè i hi } \\
\text { è hé hè i hi }\end{array}$ \\
\hline Transcription $\mathrm{N}^{\mathrm{o}} 3$ : & $\begin{array}{l}\text { grave } \\
\text { medium } \\
\text { aigu }\end{array}$ & $\begin{array}{l}\text { a ay é hé } \\
\text { a é ha hay i } \\
\text { é hé hi }\end{array}$ \\
\hline
\end{tabular}

Bien qu'il s'agisse dans les trois cas de Chants du vautour, leur texte diffère en partie, ce qui est un autre trait saillant de cette musique qui paraît monotone à la première écoute mais révèle néanmoins d'importantes variations de détail.

Dans cette liste, $e ́$ et $\grave{e}$ sont prononcés comme en français, $h$ comme en anglais. 
Allegro assai 244 . Rubato $\langle R\rangle$ : Respiration perceptible
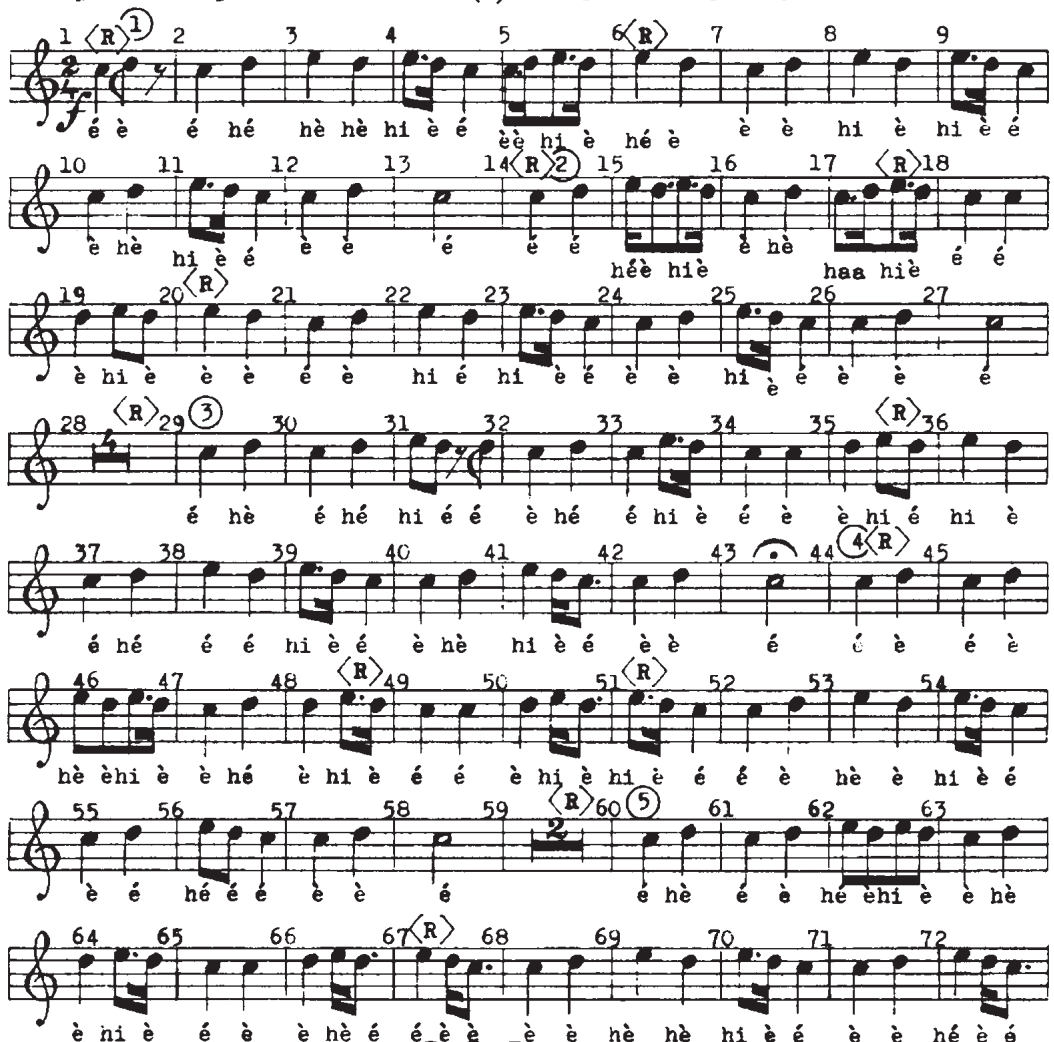

è hi è é è è hè é é è

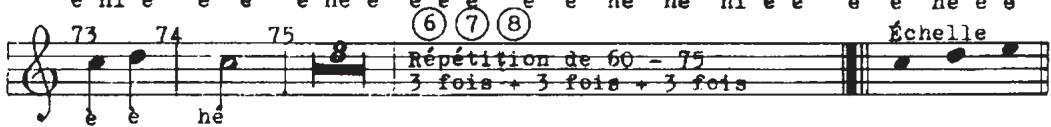

Transcription $\mathrm{N}^{\mathrm{o}} 1$.

Il n'est guère possible ici de transcrire minutieusement tous les Chants $d u$ vautour enregistrés chez les Xetá, sans compter que cet exercice ne nous apprendrait pas plus, au demeurant, que notre sélection de trois chants. Alors que ceux-ci se ressemblent du point de vue de leurs éléments mélodiques et rythmiques, leur structure est si imprécise et variable qu'il est impossible d'en dégager une forme standard. C'est pourquoi il semble opportun de faire ressortir plutôt les traits saillants des enregistrements, sauf dans les cas où nous sommes en mesure de formuler des considérations théoriques générales. Cette dernière remarque s'applique avant tout aux chants répétées par le même individu. Dans ces cas, la comparaison de deux versions permet de découvrir quels facteurs sont suffisamment significatifs aux yeux du chanteur xetá pour qu'il considère deux versions du Chant du vautour comme étant identiques. 
De toute évidence, les Xetá reconnaissent deux niveaux d'identité. Le premier est le niveau général leur permettant de déterminer la catégorie de chant, par exemple un Chant du vautour. Pour l'instant, nous ne sommes pas à même de répondre aux questions liées à ce niveau. Le second niveau est spécifique, en ce sens qu'il permet aux Xetá de reconnaître l'exécution du chant «d'après la voix» de quelqu'un. Commençons par l'analyse de ce dernier aspect. Voici la manière dont sont introduits deux enregistrements datés de février 1961: tout d'abord, «Tuca interprète le Chant du vautour d'après la voix de Mwatxagwi, frère de Niango», suivi immédiatement par: "le même est répété». Le même homme répète donc le même chant, et comme il faut exclure ici la possibilité que le chanteur ait omis quelque détail de la seconde version, toute différence à déceler entre les deux interprétations doit être considérée comme insignifiante aux yeux des Xetá.

Les deux chants en question figurent dans les transcriptions $\mathrm{N}^{\text {os }} 4$ et 5 . Passons maintenant à leur comparaison et signalons-en similitudes et différences. La phrase musicale 1 de la version I comporte 7 pulsations, tandis que celle de la version II en comporte 12. D'une manière générale, et en faisant abstraction des différences minimes, nous constatons ceci: phrase 2 de la version II $=2$ (phrase 1 de la version I).

Dans les phrases suivantes des deux versions, d'autres différences apparaissent, qui consistent partiellement en variations rythmiques assez marquées et fréquentes pour autoriser le constat que voici: les deux versions sont au moins aussi différentes l'une de l'autre que n'importe lesquelles des deux versions du Chant du vautour interprétée par deux chanteurs différents. Considérons les différences tout d'abord.

La version I est constituée de cinq phrases musicales et de 56 pulsations, alors que la version II comporte six phrases musicales et 70 pulsations. Les phrases musicales elles-mêmes diffèrent: en tolérant quelques légères variations d'ordre rythmique ou mélodique, elles peuvent être regroupées en quatre types principaux ${ }^{2}$ :

$$
\begin{array}{ll}
\text { Type A : } & \text { version I, phrase } 1 \\
& \text { version II, phrase } 5 \\
\text { Type B : } & \text { version I, phrase } 2 \\
\text { Type C : } & \text { version I, phrase } 3,5 \\
& \text { version II, phrase 2,4 } \\
\text { Type D : } & \text { version I, phrase 4 } \\
& \text { version II, phrase } 1,3,6
\end{array}
$$

Pour les deux versions, on obtient ainsi la structure suivante:

\section{Version I : A B C D C Version II : D C D C A D}

Mis à part le fait que la séquence CDC apparaît dans les deux, leur structure respective ne pourrait guère être plus dissemblable.

Pour les types A et D, nous n'avons pas pris en considération l'écart d'un demi-ton. 
Allegro 138. Rubato

(1)

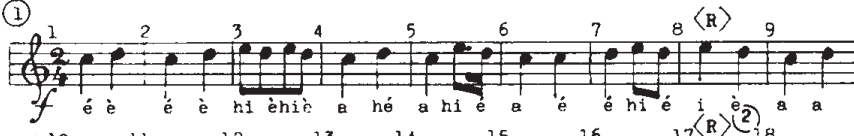

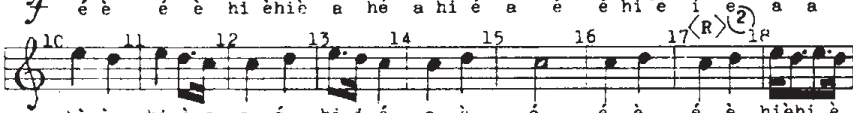

hè è hi è a é hi i é $\langle$ è é é è é è hièni

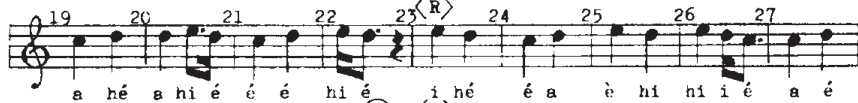

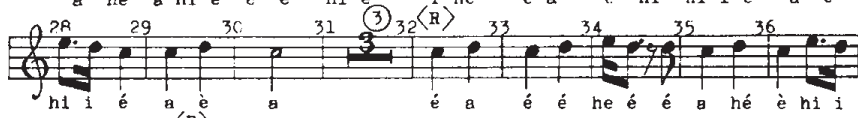

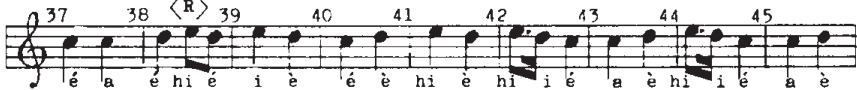
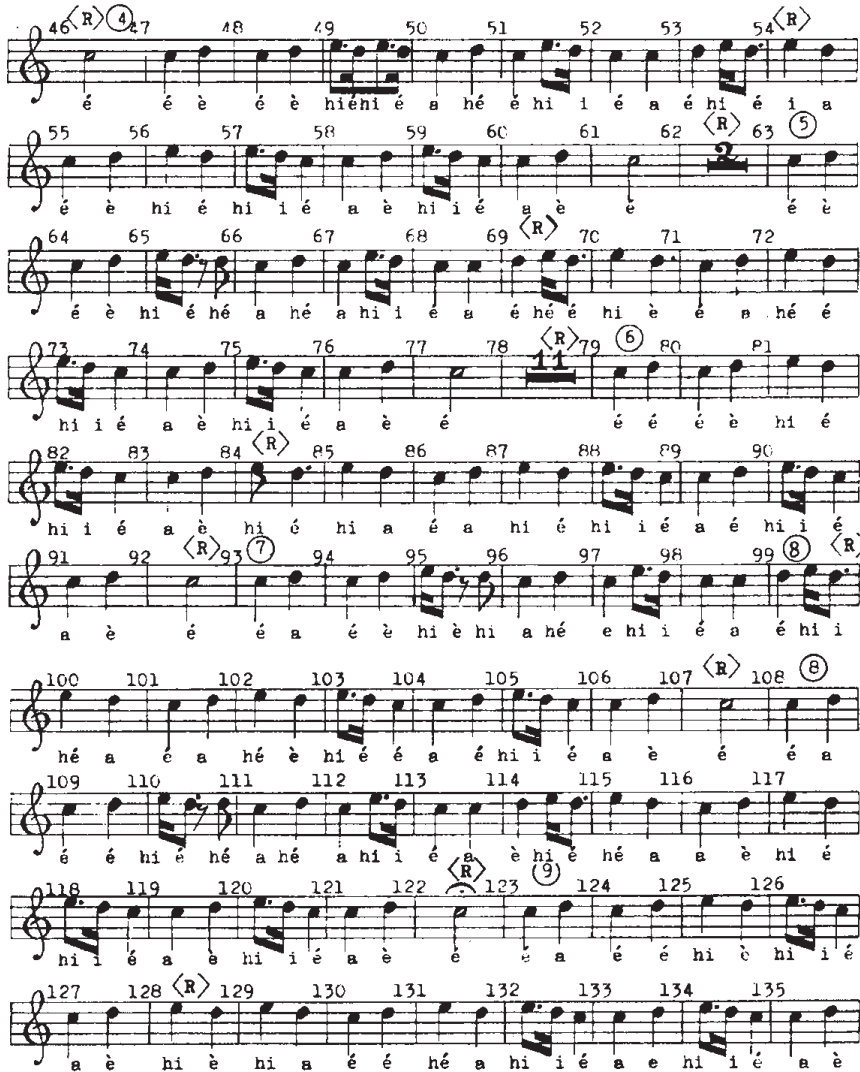

Transcription $\mathrm{N}^{\circ} 2$. N.B. A partir de la mesure 10 , la hauteur de son s'élève graduellement pour atteindre un demi-ton à la fin de la pièce.
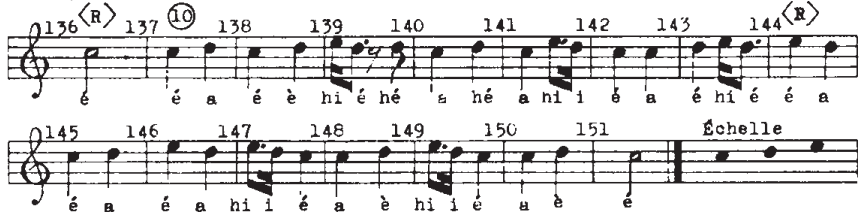
Quant aux similitudes les plus marquantes, on constate que les deux versions contiennent les mêmes quatre tons; que leur première phrase musicale comporte une échelle constituée d'intervalles de deux tons et d'un demi-ton, alors que le reste se fonde sur une échelle de deux tons et deux demi-tons; qu'à l'exception du type B, elles contiennent les mêmes types de phrases musicales, bien que celles-ci soient agencées différemment; que le tempo des deux pièces est semblable: 150 et 148 ; que les éléments rythmiques sont les mêmes, et que le mode d'exécution en falsetto, avec tension extrême des cordes vocales, se rencontre dans les deux cas; enfin, que le texte est identique.

Le fait que les deux phrases initiales se fondent sur une échelle différente de celle des autres phrases semble indiquer que ce phénomène est à la fois intentionnel et significatif du point de vue musicologique.

La transcription et l'analyse d'autres Chants du vautour nous amènent alors à la conclusion suivante: quand un Xetá dit qu'il interprète un chant dans la voix de quelqu'un, il ne veut pas dire que cette interprétation est une réplique plus ou moins fidèle de la manière dont la personne évoquée l'a exécuté. Certaines particularités, l'emploi du staccato, l'évitement délibéré d'une des notes d'une phrase musicale bien connue, certains moyens verbaux non utilisés par les autres interprètes, la légère modification de la hauteur par un demi-ton apparaissent comme autant de tentatives pour imiter les particularités individuelles de la personne évoquée, mais le chanteur garde une grande liberté quant aux propriétés tant structurelles que mélodiques et rythmiques de la pièce. D'autre part, l'agencement des phrases musicales ne semble revêtir que peu d'importance pour le chanteur xetá.

Étant donné nos lacunes sur la musique des Xetá, il serait abusif d'affirmer que le fait de chanter «d'après la voix» de quelqu'un fait partie d'un rituel plutôt qu'il ne représente un art, mais à considérer les deux exemples et, à un degré non moindre, la répétition ad infinitum de quelques phrases musicales, on a l'impression que l'exécution d'un Chant du vautour représente pour les Xetá une sorte de rite de réciprocité. L'identité spécifique d'un chant semble être fictive plutôt que réelle.

Parmi les vingt-trois versions recueillies du Chant $d u$ vautour, il y a deux duos et deux trios. Leur analyse, effectuée sur la base d'une transcription rendant compte du moindre détail, fait apparaître d'autres caractéristiques des duos xetá: la voix II commence au moment où la voix I a déjà exécuté sept mesures; le même phénomène s'observe au début de chaque phrase musicale, la voix II étant toujours en retard; les voix sont décalées par une, deux, six, sept ou neuf mesures; on relève un demi-ton de différence entre les tonalités des voix I et II, l'échelle de la première contenant trois intervalles d'un demi-ton, celle de la seconde six; il ne semble pas y avoir de rapport précis entre les voix I et II, en dehors du fait qu'elles ne sont presque jamais complètement parallèles ou identiques; les chanteurs semblent s'efforcer d'éviter à tout prix le chant à l'unisson, ce qui apparaît clairement dans les trios où chaque interprète se lance à un moment différent et exécute une mélodie différente sur un texte différent, pour terminer à un moment différent. 

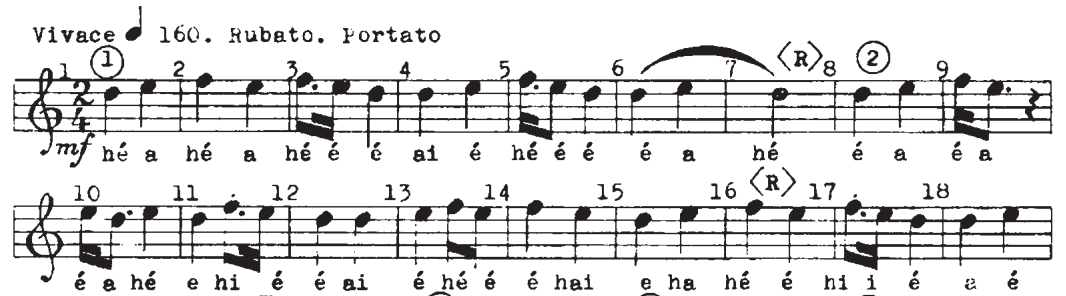

19
20
(3)

(5)

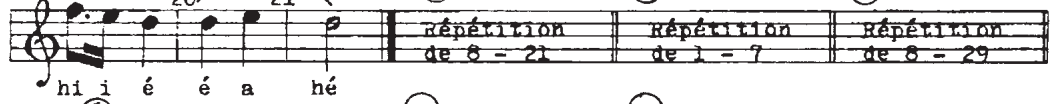

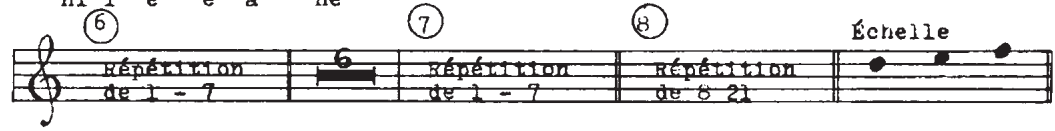

Transcription 3.

Allegrn vivace 148

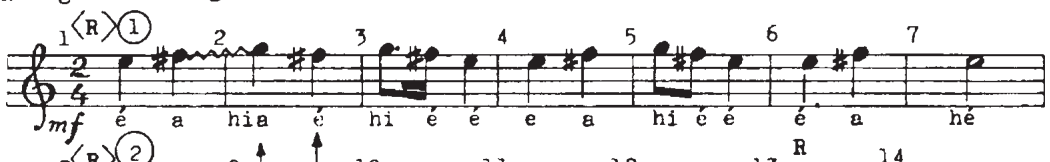

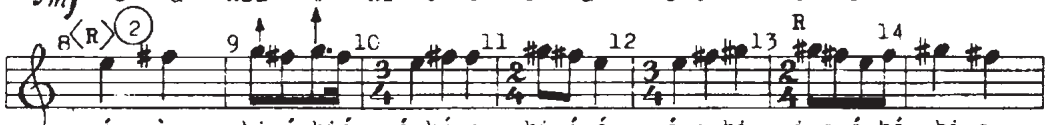
$\int$ é hi é hié é hé a hi é é é a hi i a é hé hi a (1)

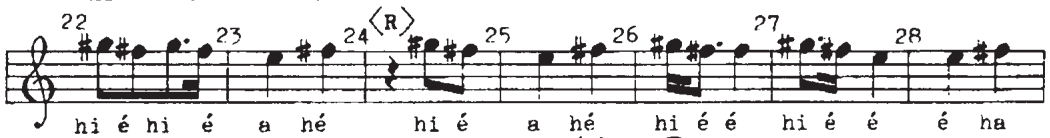

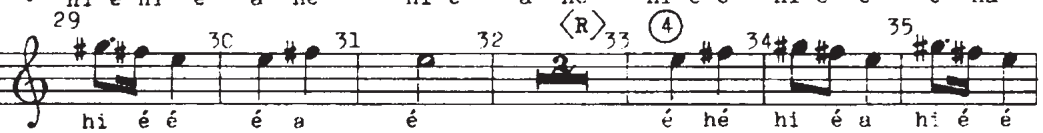

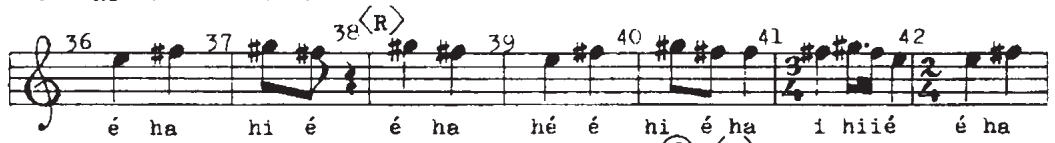

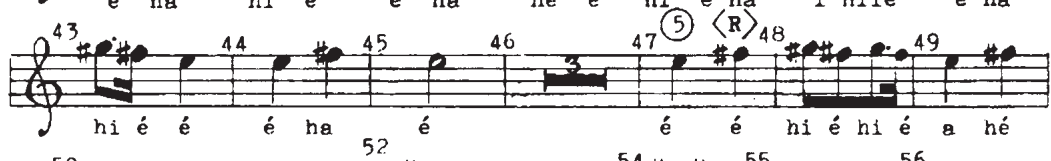
$\frac{\int^{50}}{h 1}$ Echelle

Echelle

Transcription 4, Variation I. 
Cette forme de chant semble être assez répandue parmi les Indiens d'Amérique du Sud. Je l'ai rencontrée chez les Xavante qui saluent le retour d'un parent ou d'un ami longtemps absent par un chant où chaque homme et chaque femme interprète son air personnel sans tenir compte du rythme, de la hauteur ou des paroles des chants des autres. Un phénomène similaire semble être le chant dit akia des Suya, décrit par Seeger (1977: 40-41), et nous suggérons l'hypothèse que les Xetá le leur ont emprunté. Par comparaison avec le chant des Xavante - et probablement des Suya - les duos et trios des Xetá paraissent cependant plus élaborés: sans observer de règles strictes, les Xetá semblent néanmoins en connaître, et leurs voix ne sont pas totalement indépendantes les unes des autres.

Wladimir Kozák, l'un des participants aux missions de recherche que l'Université de Paraná a entreprises chez les Xetá au moment des premiers contacts, a tourné un film $16 \mathrm{~mm}$ d'une durée de huit heures. Nous avons eu la possibilité de visionner ce beau document réalisé avec beaucoup de soin. La bobine No3 montre un rite en relation avec le vautour urubu (Coragyps atratus), dont voici une description détaillée basée, non seulement sur le film lui-même mais encore sur les communications personnelles de son réalisateur que nous avons rencontré quelques mois avant sa mort.

Un groupe de Xetá a fléché un urubu. Dans une petite clairière, ils ont érigé une structure en bois composée d'une poutre d'une longueur de quatre mètres environ, fixée à deux mètres du sol dans la fourche naturelle d'un tronc d'arbre et reliée à un autre tronc au moyen d'une longue bande d'écorce. Deux Indiens sortent de la forêt, apportant l'oiseau mort. L'un d'eux tient une hache de pierre à la main, l'autre une arme faite de bois dur, semblable à une pêle ou à une large rame au manche relativement court. Cette arme - que nous avons retrouvée dans la collection d'objets assemblés par Kozák - était surtout utilisée pour la guerre. S'il semble assez naturel qu'un Indien se promène avec une hache de pierre, le port d'une arme de combat peut paraître incongru, et nous pensons qu'elle servait de symbole pour rehausser l'acte rituel.

Les deux Indiens se dirigent vers la structure en bois sur laquelle ils attachent l'oiseau au moyen de deux cordes fixées aux ailes. Ensuite, ils regardent le vautour pendant quelque temps, puis s'en vont. Bientôt, d'autres Indiens arrivent, un homme, une femme et deux ou trois enfants; ils s'asseyent sur un tronc d'arbre devant l'oiseau et l'épient. Peu après, ils repartent, tandis que les deux chasseurs retournent, délient le vautour, lui arrachent les pennes et les rémiges, puis rattachent les ailes et replacent l'oiseau dans la position originelle sur la structure en bois. Ils s'en vont en laissant le vautour là, sans plus le toucher, et se dirigent vers les cabanes où les hommes, appuyés contre les poteaux de leurs demeures, entonnent le Chant de l'urubu. M. Kozák a appris que ce chant ne devait pas être exécuté pendant la nuit.

De toute évidence, ce que nous venons de décrire fait partie d'un rite dont la signification, hélas, demeure obscure, probablement à jamais. Il se peut que le vautour ait été un totem, mais il est également possible qu'il ait une autre signification. L'arrachage de ses pennes pourrait être un acte symbolique d'ordre 

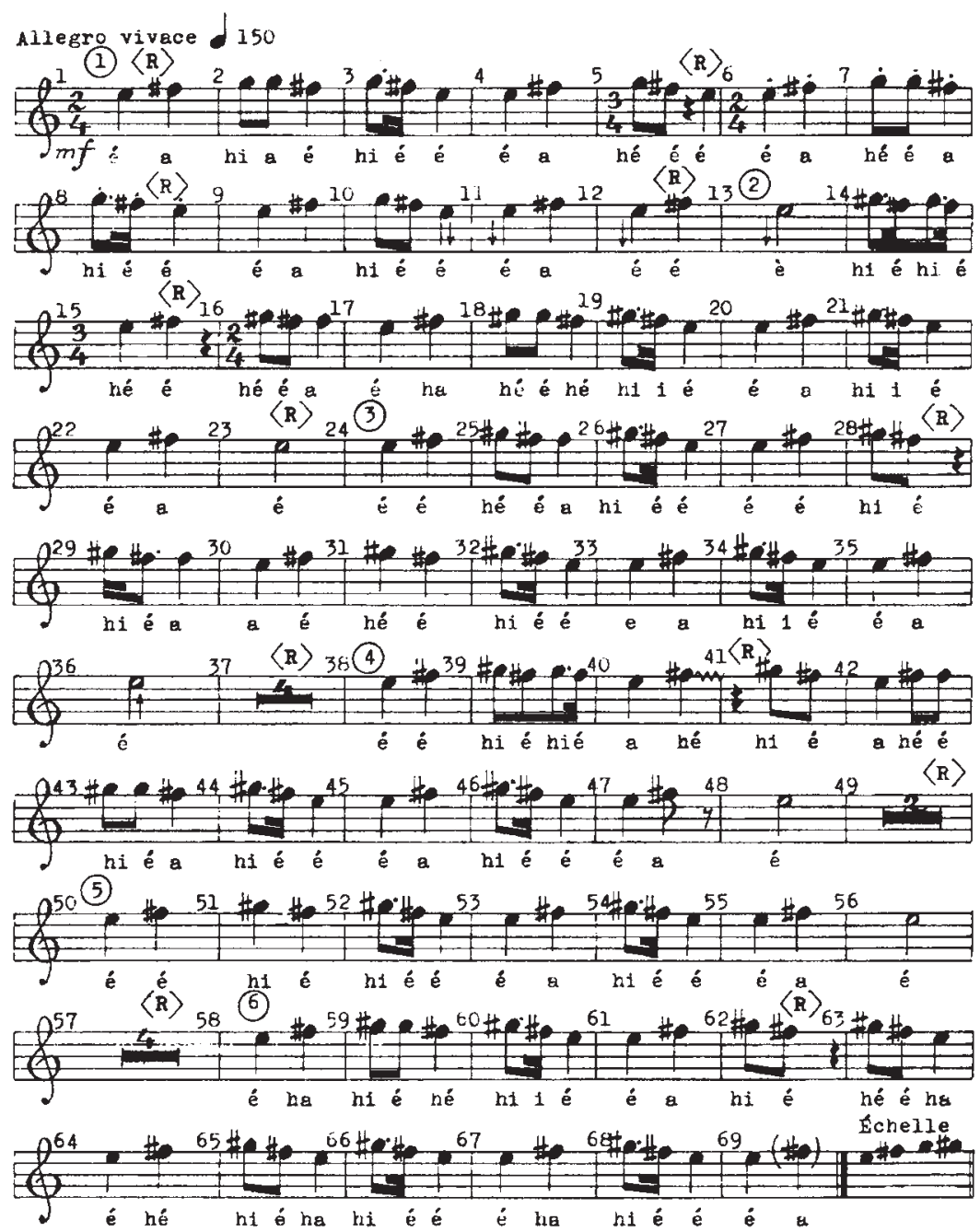

Transcription $\mathrm{N}^{\mathrm{o}}$ 5, Variation II.

métonymique pour montrer que l'oiseau n'était pas seulement mort et attaché, mais encore à jamais incapable de s'envoler (Leach 1976: 21). D'autre part, il pourrait s'agir d'une mesure pratique destinée simplement à préserver les grandes plumes utiles, bien que dans ce cas-là, celles-ci auraient pu être arrachées avant que l'oiseau ne fût attaché pour la première fois.

Nos enregistrements de la musique xetá semblent relever d'une série plus étendue de chants assez semblables. Au terme de la description, de la transcription partielle et de l'analyse d'une catégorie majeure, celle des Chants $d u$ 

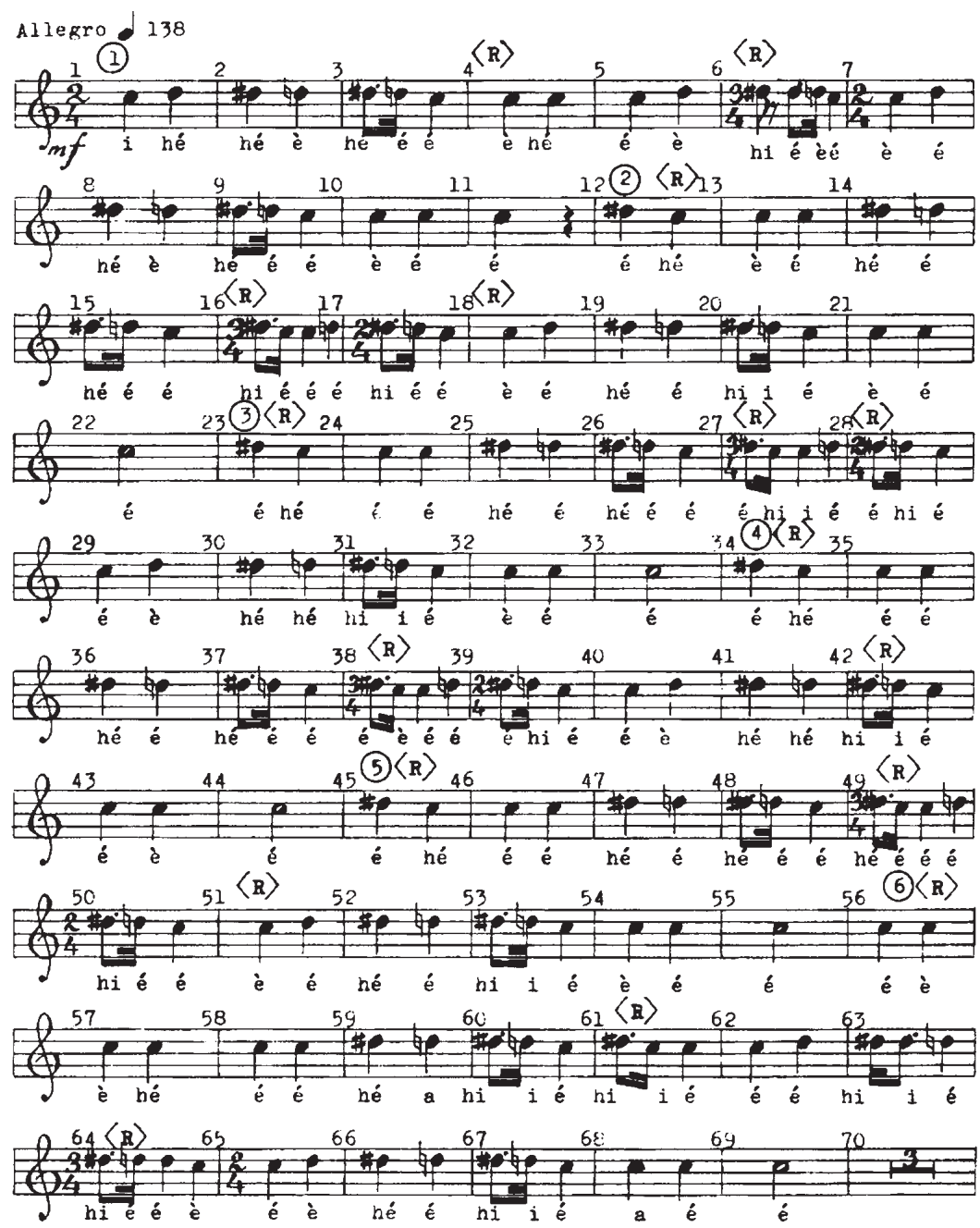

Transcription $\mathrm{N}^{\circ} 6$.

vautour, et en suivant la séquence des enregistrements, il nous reste à appliquer la même démarche à une autre catégorie qui est celle des Chants des étoiles.

La transcription $\mathrm{N}^{\circ} 6$ en donne un exemple interprété par Ajatukã «dans la voix de Mboatxagwi». Son échelle est à nouveau très réduite, contenant deux tons et deux demi-tons au début. Or, dans la deuxième partie s'effectue un changement de tonalité à peine perceptible, si bien qu'à la fin, on en arrive à deux tons et un demi-ton. La question de savoir s'il s'agit là d'un changement dont le chanteur serait inconscient est sujette à débat. La modification graduelle de tous les tons d'un chant, si répandue dans la musique amérindienne, pourrait 
(7) $\langle$ B $\rangle$
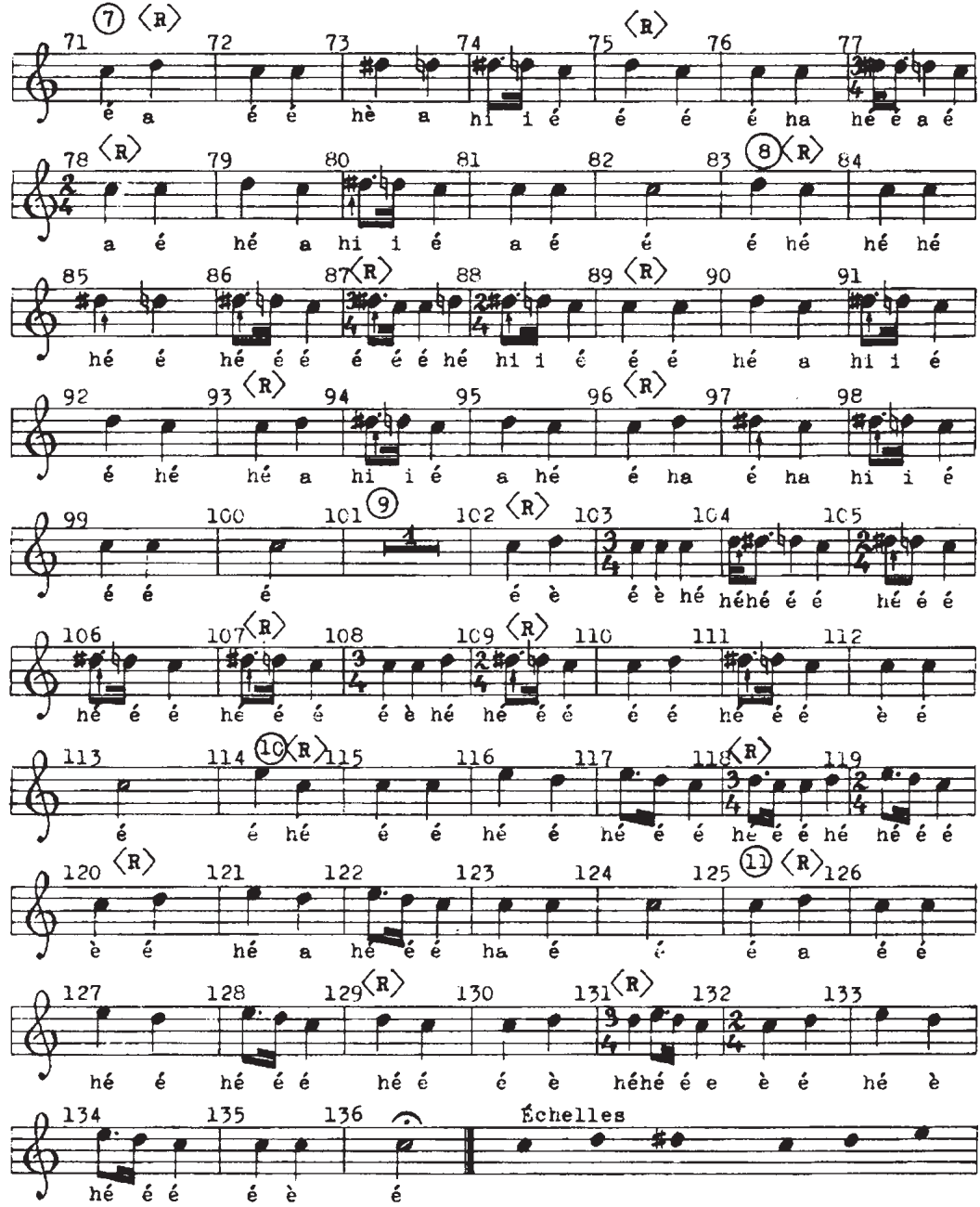

Transcription $\mathrm{N}^{\mathrm{o}} 6$ (suite).

résulter d'une ouïe imparfaite, mais à notre avis la modification d'un seul ton est intentionnelle. Si cette hypothèse s'avère juste, le chant en question revêt un intérêt théorique certain, car le changement graduel de la tonalité serait alors un trait saillant de la musique des Xetá, mais peu fréquent dans la musique des Indiens du Brésil.

Il serait déçu, l'auditeur qui, las d'entendre les mêmes phrases musicales (ou presque) de diverses variantes du Chant du vautour, s'attendrait à rencontrer du nouveau dans la composition du Chant des étoiles. Les phrases musicales de la transcription $\mathrm{N}^{\mathrm{o}} 6$ se distinguent vaguement de celles des autres notations, 
mais ces différences sont si légères et les similitudes si marquées que l'auditeur non indien a de la peine à déterminer quel chant il est en train d'entendre. Nous croyons pouvoir renoncer ici à la comparaison détaillée telle que nous l'avons effectuée pour la catégorie des Chants du vautour : son résultat serait à peu près le même. Mais il convient de rappeler que les différences d'une pièce à l'autre se résument à des modifications presque microscopiques et à peine perceptibles que l'auditeur non xetá a beaucoup de peine à reconnaître.

Les bandes magnétiques contiennent toute une série de chants introduits par l'orateur dans la langue des Xetá. Bien qu'il nous soit impossible de comprendre le sens des paroles, nous avons soigneusement comparé les titres pour découvrir s'il y avait répétition de certains mots. Nous avons découvert que le terme de nakutnak figure dans onze chants, le plus souvent comme mot final. Nous avons comparé les mélodies de ces chants, et il s'est avéré qu'elles forment un groupe à part dans lequel, à l'instar de la catégorie des Chants $d u$ vautour ou des étoiles, une ligne mélodique est constituée des mêmes éléments mélodiques et rythmiques, avec quelques rares variations, mis à part l'agencement des phrases musicales. Par la suite, nous avons sélectionné les autres chants ayant la même mélodie pour constituer ainsi une nouvelle catégorie de vingt-six chants, formant un troisième genre vocal majeur des Xetá.

Vers la fin de la dernière bande, nous avons rencontré un chant différent de tous les autres, donné dans la transcription $\mathrm{N}^{\mathrm{o}}$ 7. Comme la plupart des pièces xetá, il est constitué de trois notes, mais les intervalles portent sur quatre et trois demi-tons. Il contient huit phrases musicales plutôt semblables, la seule particularité étant que deux d'entre elles - les phrases 5 et 8 - sont des formes abrégées de la phrase 1 . Le texte se construit presque exclusivement sur le phonème é.

Il est évident que notre aperçu de la musique vocale des Xetá, en partie conjectural, n'est nullement exhaustif. Il existait sans doute des traits dans le système musical des Xetá que nous ne découvrirons jamais. Toutefois, le peu de données disponibles constitue déjà un système musical dont voici le modèle en guise de conclusion.

\section{Le modèle conceptuel de la musique xetá}

Pour interpréter un chant à la manière des Xetá, il faut connaître le modèle que voici, bien que celui-ci soit probablement inconscient et certainement non verbalisé. Selon la classification de Schaefer et Novak, il appartient à la catégorie des «modèles observés» (beobachtete Modelle). Or, s'il s'agit d'un modèle fondé exclusivement sur notre propre observation et nos propres hypothèses, il n'en était pas moins, pour le Xetá qui le suivait, une réalité issue de la tradition (Schafer et Novak 1972: 24). 


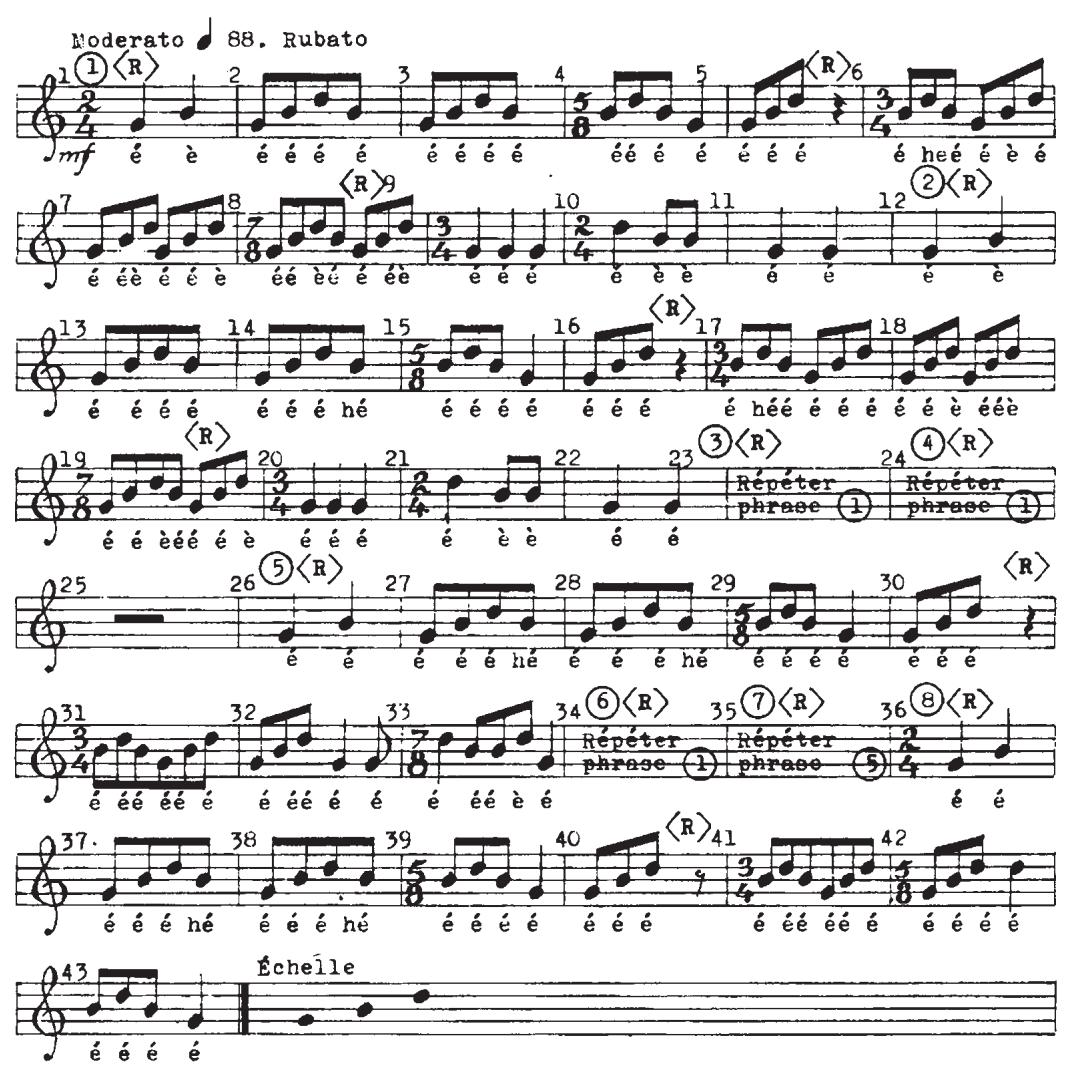

Transcription $\mathrm{N}^{\mathrm{o}} 7$.

1 - Classification des pièces vocales. Les catégories générales des pièces vocales sont peu nombreuses: Chants du vautour et des étoiles, puis un troisième type de chants dont nous ne comprenons pas la dénomination. Mais puisque chaque chant peut être interprété «dans la voix» de divers individus, on peut considérer ces variantes comme des sous-catégories.

2 - Technique de chant. Elle entraîne une forte tension des cordes vocales et requiert un effort considérable. La plupart des chants sont interprétés legato, mais il existe aussi quelques rares pièces en staccato.

3 -Échelle. Si tous les chants sont tritoniques, on rencontre néanmoins six tons, en tenant compte des modulations graduelles et légères - presque imperceptibles - dans un sens ascendant ou descendant. Des doutes subsistent quant à la question de savoir si le chanteur effectue consciemment ces modulations, mais il est probable que non. Dans une seule transcription $\left(\mathrm{N}^{\mathrm{o}} 6\right)$, un phénomène différent apparaît: seule une des trois notes est altérée d'un demi-ton dans le sens ascendant, ce qui ne peut de toute évidence pas être attribué à l'ouïe 
imparfaite; dans ce cas, on a donc affaire à une altération délibérée. Quant au nombre de tons, on obtient ainsi les possibilités suivantes: a) en général, trois tons; b) dans le cas d'une modification graduelle et probablement inconsciente de tous les tons: six tons; c) dans le cas d'une modification probablement consciente d'un ton seulement: quatre tons.

Les intervalles portent généralement sur deux tons et un ou deux tons et deux demi-tons, mais nous rencontrons aussi des intervalles de quatre et trois demi-tons, l'échelle complète d'une pièce pouvant ainsi être: $2+1=3$ demitons; $2+2=4$ demi-tons; enfin, $4+3=7$ demi-tons.

Des glissandos et des portamentos apparaissent parfois, s'ajoutant aux nombreuses notes (intermédiaires) qui composent le chant.

4 - Ligne mélodique. Les possibilités de variations sonores étant limitées par l'échelle essentiellement tritonique, la ligne mélodique peut difficilement être autre chose qu'une simple ondulation. Cette tendance est renforcée par le fait que les Xetá ne semblent pas apprécier les sauts mélodiques. Quand nous entendons, par exemple, la note la plus grave, nous pouvons être sûrs que la suivante sera la moyenne puisque tant le saut vers la troisième, la plus aiguë, que la répétition de la première sont assez exceptionnels. Bien qu'il existe quelques répétitions de notes, elles ne sont pas très répandues et ne surgissent pas plus d'une fois dans une même pièce. La musique des Xetá ignore complètement la répétition de la même note à cinq ou dix reprises, voire même plus souvent, si répandue dans les chants amérindiens (Xavante, Bororo).

Dans les pièces vocales, la première et la dernière note de la mélodie sont toujours identiques, et cela s'applique également aux phrases musicales, les formules de niveau se chiffrant toujours à zéro (Kolinski 1957: 3; 1976: 1).

5 - Rythme. Le rythme est simple ou d'une complexité moyenne, souvent rubato, probablement en raison du fait que nulle danse ne s'accompagne de musique, cette dernière n'étant ainsi pas gouvernée par une séquence temporelle précise (pulsation). Le tempo est plutôt vif; un mouvement lent - largo - ne se rencontre jamais. On entend souvent des pauses, longues et irrégulières, qui doivent probablement permettre au chanteur de détendre ses cordes vocales.

6 - Structure. D’une manière générale, les phrases musicales contiennent dix à quinze mesures se prêtant à être chantées sur une seule respiration. Les phrases diffèrent, mais ces différences sont le plus souvent si minimes qu'il est difficile de les détecter. Les phrases musicales tendent à être répétées, et il ne semble pas y avoir de règle gouvernant leur agencement, bien que l'on voie émerger parfois une structure strophique.

7 - Hétérophonie. Les duos et trios que nous avons transcrits appartiennent à la catégorie des pièces musicales que Curt Sachs a qualifiées d'hétérophoniques (1962: 185). Elles se caractérisent par une indépendance presque totale des voix, à l'exception du rythme. La seconde - et, le cas échéant, la troisième - intervient avec un retard d'un, de deux ou trois, voire même de neuf mesures par rapport à la première voix, pour exécuter une mélodie autre mais néan- 
moins semblable pouvant se situer à une hauteur différente. Les chanteurs veillent particulièrement à éviter le chant à l'unisson, ce qu'ils réalisent de la manière suivante: a) en interprétant des mélodies différentes; b) en intervenant à divers moments ; c) en adoptant des paroles différentes; d) en introduisant à des intervalles irréguliers des pauses plus ou moins longues; e) en adoptant une gamme différente. Nous ignorons les raisons de ce procédé, mais pourrions souscrire à l'hypothèse avancée par Seeger au sujet des chants akia des Suya (1977: 40-41), selon laquelle chaque interprète œuvre pour être entendu et reconnu à titre individuel.

Toutes ces restrictions ont, par la force des choses, produit un système musical limité qui interdit des initiatives d'ordre mélodique, rythmique ou structurel: c'est un véritable microcosme musical. Mais c'est là une approche ethnocentrique. Pour les Xetá, les possibilités de variation ne résident pas dans la libre transformation de la mélodie et du rythme, mais passent plutôt par des modifications de détail à peine perceptibles mais néanmoins d'une signification esthétique considérable, alors qu'ils nous paraissent négligeables. Le chanteur xetá, loin d'être l'imitateur primitif d'un répertoire fort réduit, n'avait pas non plus pour seul rôle de reproduire les chants légués par la tradition: quand il entonnait un chant «dans la voix» de quelqu'un, il se muait en compositeur, donnant une nouvelle forme aux pièces coutumières. Son art ne consistait pas à créer du nouveau, mais à élaborer les menus détails dont étaient investies les formes anciennes.

Voici ce que nous enseignent les Xetá : une nouvelle compréhension de leur art, et c'est pourquoi nous déplorons la totale disparition de leur culture.

\section{Bibliographie}

HORNBOSTEL Erich M. von \& Curt SACHS

1961 «Classification of musical instruments». Traduit de l'allemand par Anthony Baines et Klaus P. Wachmann. The Galpin Society Journal XIV: 17.

JAIRAZBHOY Nazir A.

1977 «The "objective" and subjective view in music transcription». Ethnomusicology XXI(2): 264-82.

KOLINSKI Miecyzslaw

1957 «Ethnomusicology, its problems and methods». Ethnomusicology Newsletter 10 (Middleton): 1-7.

1959 «The evaluation of tempo». Ethnomusicology III(2): 45-57.

1976 «Herndon's verdict on analysis: TABULA RASA». Ethnomusicology XX(1): 1-22.

LAMING-EMPERAIRE Annette

1964 «Les Xeta, survivants de l'âge de la pierre». Objets et Mondes IV(4): 263-64.

LAMING-EMPERAIRE Annette, MENEZES Maria José \& Margarida Davina ANDREATTA 1978 O trabalho de pedra entre os Xetá, Serra dos Dourados, Estado do Paraná. São Paulo: Universidade de São Paulo (Fundo de Pesquisas do Museu Paulista). 


\section{LEACH Edmund}

1976 Culture and Communication, the Logic by which Symbols are Connected. An Introduction to the Use of Structuralist Analysis in Social Anthropology. Cambridge: Cambridge University Press.

LOUREIRO FERNANDES José

1959 «Os índios da Serra dos Dourados [os Xetá]». In Anais da III. Reunião Brasileira de Antropologia, Recife, p. 895-98.

1961 «Le peuplement du Nord-Ouest du Paraná et les Indiens de la "Serra dos Dourados"». Boletim Paranaense de Geografia 2-3: 80-92.

1962 «Os índios da Serra dos Dourados. Estado atual das pesquisas». Bulletin of the International Committee on Urgent Anthropological and Ethnological Research 5: 27-45.

MOORE Michael

1974 «The Seeger Melograph Model C ». In: Selected Reports in Ethnomusicology II (1): 1-13.

NETTL Bruno

1964 Theory and Method in Ethnomusicology. New York/London: The Free Press/Collier Macmillan.

1972 Music in Primitive Culture. Cambridge, Mass.: Harvard University Press.

REICHEL-DOLMATOFF Gerardo

1973 Desana. Le symbolisme universel des Indiens Tukano du Vaupès. Paris: Gallimard.

\section{SACHS Curt}

1962 The Wellsprings of Music. New York: Da Capo Paperbacks.

SCHAEFER H. \& P. NOVAK

1972 «Anthropologie und Biophysik». In: Neue Anthropologie. Hans-Georg Gadamer \& Paul Vogler Hg., Band I: Biologische Anthropologie. München: DTV, p. 22-58.

\section{SEEGER Anthony}

1977 «Porque os índios Suyá cantam para as suas irmãs?» In: Arte e Sociedade, Ensaios de Sociologia de Arte, organizado por Gilberto Velho. Rio de Janeiro: Zahar Editores, p. 39-63. 